\title{
Replication kinetics and persistence of a conditionally live attenuated SIV (SIVrtTA) in vivo confers protection against homologous and heterologous wild-type challenge in macaques
}

\author{
Neil Berry ${ }^{1 *}$, Maria Manoussaka ${ }^{3}$, Claire Ham, Deborah Ferguson ${ }^{1}$, Mark Page ${ }^{1}$, Richard Stebbings ${ }^{1}$, Atze Das ${ }^{2}$,
} Martin Cranage ${ }^{3}$, Neil Almond ${ }^{1}$, Ben Berkhout ${ }^{2}$

From Frontiers of Retrovirology: Complex retroviruses, retroelements and their hosts Cambridge, UK. 16-18 September 2013

\section{Background}

Vaccination with live attenuated SIV confers potent protection against wild-type SIV challenge in the SIV/ macaque model of HIV. Although safety concerns preclude direct application of this vaccine approach in humans, a clearer understanding of the mechanistic processes at play may lead to better HIV vaccines. A novel live attenuated SIVmac239 $\Delta$ nef vaccine (SIVrtTA), dependent on doxycycline (dox) administration for replication, has been evaluated for its ability to protect in vivo against independent homologous and heterologous pathogenic wild-type (wt) SIV challenges, and the role of vaccine persistence assessed. We recently demonstrated immunophenotyping of SIVrtTA vaccinees prior to wt challenge drives global polarisation of a $\mathrm{T}$ cell memory phenotype, particularly in the $\mathrm{CD} 4$ population, toward an effector memory response.

\section{Materials and methods}

Initially, two groups of six Indian rhesus macaques (RM) were vaccinated with SIVrtTA with an oral doxycycline dosing regime for 6 months. In Group A, doxycycline was stopped 8 weeks before homologous wild-type SIVmac239 challenge but continued in Group B. Separately, Mauritian cynomolgus macaques $(\mathrm{CM})$ were vaccinated with SIVrtTA in three groups (Grp A; 20 wks dox-on, Grp B; 3 wks dox-on, 17 wks dox-off; Grp C; 3 wks dox-on) and challenged with the heterologous wild-type SIVsmE660. Kinetics of vaccine virus replication and

'Virology, NIBSC, South Mimms, Hertfordshire, UK

Full list of author information is available at the end of the article superinfection resistance outcomes were determined by sensitive and specific molecular and virological assays and detailed immunological studies performed.

\section{Results}

Following wild-type challenge with either SIVmac239 or SIVsmE660, all SIVrtTA vaccinees exhibited significantly lower levels of breakthrough virus which in 2/8 SIVrtTA vaccinees challenged with SIVmac239 and 10/18 challenged with SIVsmE660 were protected from detectable wt virus. While the kinetics of vaccine virus replication differed between species, in Indian RM enhanced control of wild-type virus was correlated with a transitory shoulder of sustained plasma viral RNA load in the period up to 100 days post-vaccine delivery. On the day of challenge, a high frequency of circulating CD8+ $\mathrm{T}_{\mathrm{EM}}$ was associated with protection but was not necessarily essential. In Mauritian CM there was a clear correlation between persistence of the vaccine virus and protection with $6 / 6$ protected against detectable SIVsmE660 infection after 20 weeks vaccination (dox 'on'), with $1 / 6$ protected after 3 weeks vaccination (dox 'on') and 3/6 dox 'on' for 3 weeks followed by 17 weeks dox 'off'.

\section{Conclusions}

Together, these data suggest a maturation of protection with longer periods of vaccination is beneficial, even when the vaccine-virus is apparently 'switched-off'. Since the genetics and antigenic relatedness of vaccine and challenge virus does not appear to predict the outcome of these vaccine studies, the kinetics and relative persistence of vaccine virus replication in contributing to 
adaptive and innate immune responses are being evaluated. The outcome of these studies will inform rational HIV/AIDS vaccine design

\section{Authors' details}

${ }^{1}$ Virology, NIBSC, South Mimms, Hertfordshire, UK. '2Laboratory of Experimental Virology, Department of Medical Microbiology, Centre for Infection and Immunity Amsterdam, Academic Medical Center of the University of Amsterdam, Amsterdam, The Netherlands. ${ }^{3}$ Division of Cellular and Molecular Medicine, St. George's, University of London, London, UK.

Published: 19 September 2013

doi:10.1186/1742-4690-10-S1-P5

Cite this article as: Berry et al: Replication kinetics and persistence of a conditionally live attenuated SIV (SIVrtTA) in vivo confers protection against homologous and heterologous wild-type challenge in macaques. Retrovirology 2013 10(Suppl 1):P5.

Submit your next manuscript to BioMed Central and take full advantage of:

- Convenient online submission

- Thorough peer review

- No space constraints or color figure charges

- Immediate publication on acceptance

- Inclusion in PubMed, CAS, Scopus and Google Scholar

- Research which is freely available for redistribution

Submit your manuscript at www.biomedcentral.com/submit
C Biomed Central 